\title{
Planar Hall effect and magnetic anisotropy in epitaxially strained chromium dioxide thin films
}

\author{
S. T. B. Goennenwein ${ }^{a)}$ \\ Kavli Institute of NanoScience, Faculty of Applied Sciences, Delft University of Technology, 2628 CJ Delft, \\ The Netherlands and Walther-Meissner-Institut, Bayerische Akademie der Wissenschaften, Garching, \\ 85748 Munich, Germany \\ R. S. Keizer \\ Kavli Institute of NanoScience, Faculty of Applied Sciences, Delft University of Technology, 2628 CJ Delft, \\ The Netherlands \\ S. W. Schink \\ Walther-Meissner-Institut, Bayerische Akademie der Wissenschaften, 85748 Garching, Germany \\ I. van Dijk and T. M. Klapwijk \\ Kavli Institute of NanoScience, Faculty of Applied Sciences, Delft University of Technology, 2628 CJ Delft, \\ The Netherlands \\ G. X. Miao \\ MINT Center, University of Alabama, Tuscaloosa, Alabama 35487 and Physics Department, Brown \\ University, Providence, Rhode Island 02912
}

G. Xiao

Physics Department, Brown University, Providence, Rhode Island 02912

A. Gupta

MINT Center, University of Alabama, Tuscaloosa, Alabama 35487

(Received 8 September 2006; accepted 18 February 2007; published online 4 April 2007)

We have measured the in-plane anisotropic magnetoresistance of $100 \mathrm{~nm}$ thick $\mathrm{CrO}_{2}$ thin films at liquid He temperatures. In low magnetic fields $H$, both the longitudinal and the transverse (planar Hall) resistance show abrupt switches, which characteristically depend on the orientation of $H$. All the experimental findings consistently demonstrate that the magnetic anisotropy in these $\mathrm{CrO}_{2}$ thin films is biaxial. We show that the biaxial magnetic anisotropy is due to epitaxial coherency strain, and that it naturally explains the complex magnetic switching behavior reported recently in $\mathrm{CrO}_{2}$ films with thicknesses of $50 \mathrm{~nm} \leqslant d \leqslant 250 \mathrm{~nm}$. (C) 2007 American Institute of Physics.

[DOI: $10.1063 / 1.2715442]$

Half-metallic ferromagnets (HMFs) such as chromium dioxide $\left(\mathrm{CrO}_{2}\right)$ are intriguing materials. ${ }^{1}$ Their density of states is finite at the Fermi energy $E_{F}$ for one spin direction, while an insulating gap exists for the other. Accordingly, HMFs are $100 \%$ spin polarized at $E_{F}$. This makes them very attractive for the study of spin-related transport phenomena, e.g., in magnetic tunnel junctions, ${ }^{2}$ for the investigation of the spin-dependent decay of superconducting correlations, ${ }^{3,4}$ or for current-induced magnetization reversal. ${ }^{5}$

Bulk $\mathrm{CrO}_{2}$ is usually considered as a uniaxial ferromagnet with sizeable second order uniaxial contributions, the $c$ axis being the easy direction. ${ }^{6-8}$ In contrast, the magnetic properties of $\mathrm{CrO}_{2}$ thin films are more diverse. In $\mathrm{CrO}_{2}$ layers grown epitaxially on (100) $\mathrm{TiO}_{2}$ substrates, the strain resulting from the lattice mismatch makes the $b$ axis magnetically easy for layer thicknesses $d<50 \mathrm{~nm} .{ }^{9,10}$ For films with $50 \mathrm{~nm} \leqslant d \leqslant 250 \mathrm{~nm}$, a more complex magnetic behavior was observed and attributed to the superposition of different easy directions because of an inhomogeneous strain distribution across the film thickness. ${ }^{10}$ In this letter, we present anisotropic magnetoresistance (AMR) experiments, which show that the complex magnetic behavior in $100 \mathrm{~nm}$

${ }^{a)}$ Electronic mail: goennenwein@wmi.badw.de thick $\mathrm{CrO}_{2}$ films can be understood in terms of biaxial magnetic symmetry. We observe abrupt switches in the AMR, which sensitively depend on the angle enclosed between the current direction and the external, in-plane magnetic field. From the fields at which these switches take place, we conclude that the magnetic anisotropy in the film plane is biaxial. Upon taking into account the magnetic anisotropy contributions due to epitaxial coherency strain, the experimentally observed switching fields can be modeled quantitatively. This shows that crystalline strain qualitatively alters the magnetic anisotropy of $\mathrm{CrO}_{2}$ thin films.

The $100 \mathrm{~nm}$ thick, single crystalline $\mathrm{CrO}_{2}$ films are grown by chemical vapor deposition on (100)-oriented $\mathrm{TiO}_{2}$ substrates. ${ }^{9,10}$ The films are patterned into $50 \times 200 \mu \mathrm{m}^{2}$ Hall bar structures using optical lithography and wet chemical etching. For the magnetotransport experiments, the samples are mounted in a superconducting magnet system and the sample temperature is stabilized using a variable temperature insert. The magnetic field $\mathbf{H}$ is applied in the film plane. The angle $\phi$ between $\mathbf{H}$ and the current density $\mathbf{j}$ can be freely adjusted by means of a rotateable sample stage.

We simultaneously record both the resistance parallel to the current direction, $\rho_{\text {long }}$, and the resistance perpendicular to $\mathbf{j}, \rho_{\text {trans }}$. Figure 1 shows the magnetoresistance of a $\mathrm{CrO}_{2}$ Hall bar at $5 \mathrm{~K}$, for an angle $\phi=62^{\circ}$ between $\mathbf{H}$ and the 

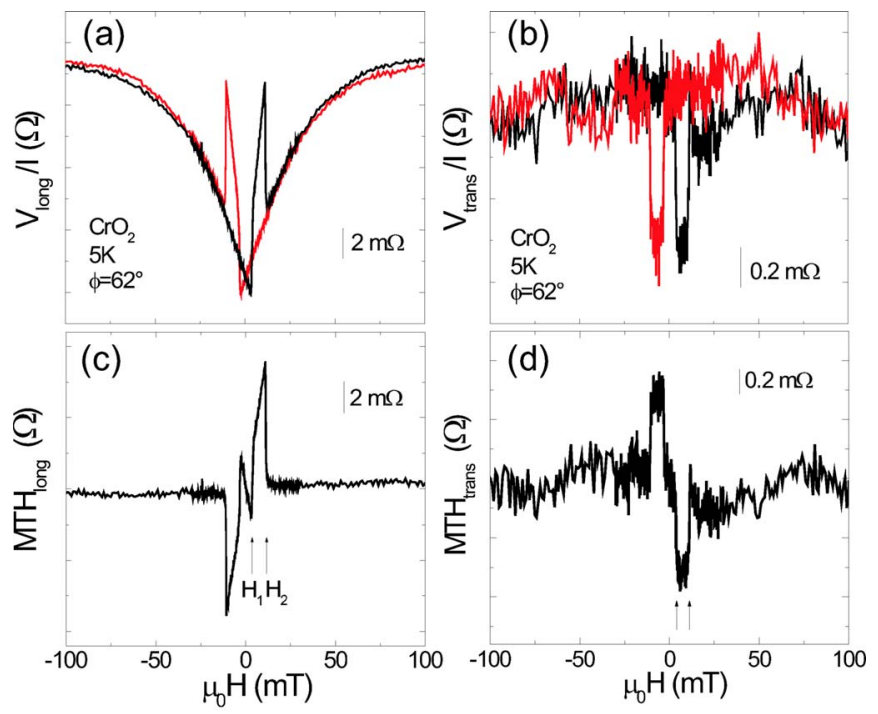

(e)

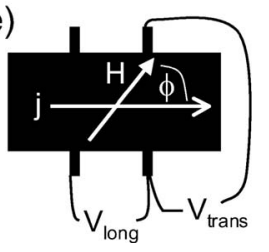

(f)

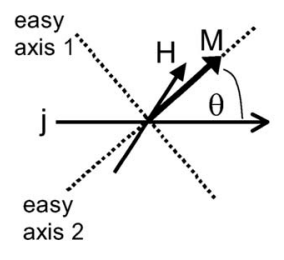

FIG. 1. (Color online) AMR of a $\mathrm{CrO}_{2}$ thin film at $T=5 \mathrm{~K}$. The data taken for the magnetic field up sweep are shown in black, the corresponding magnetic field downsweep is given in red. Both (a) the longitudinal ( $V_{\text {long }}$ $\left.\propto \rho_{\text {long }}\right)$ and (b) the transverse $\left(V_{\text {trans }} \propto \rho_{\text {trans }}\right)$ AMR components are clearly hysteretic, with steplike switches. The characteristic magnetic fields $H_{1}$ and $H_{2}$ at which these switches occur are marked by arrows. The hysteretic AMR effects can be separated from the conventional magnetoresistance background by taking the difference between magnetic field down- and up sweeps. The magneto-transport-hysteresis (MTH) curves obtained in this way are shown in panels (c) and (d) for $\rho_{\text {long }}$ and $\rho_{\text {trans }}$, respectively. The experimental setup is sketched in (e). $\mathbf{j}$ is parallel to the $\mathrm{CrO}_{2} c$ axis. As shown in (f), the orientation $\theta$ of the magnetization $\mathbf{M}$ is determined by the easy magnetic directions in the film plane for low magnetic fields. Note that $\mathbf{j}, \mathbf{H}$, and $\mathbf{M}$ all lie within the film plane.

current along the $\mathrm{CrO}_{2} c$ direction. Both $\rho_{\text {long }}$ and $\rho_{\text {trans }}$ are hysteretic as expected for a ferromagnet. Moreover, abrupt steps in the magnitude of $\rho_{\text {long }}$ and $\rho_{\text {trans }}$ are present at low $\mathbf{H}$. These are due to abrupt magnetization reorientations, as will be discussed in more detail below. To separate these features from the broad, nonhysteretic magnetoresistance, we subtract the resistance measured while sweeping the magnetic field from positive to negative values (downsweep) from the data obtained while sweeping the field from negative to positive (upsweep). In the magnetotransport hysteresis (MTH) obtained in this way, only the hysteretic part of the MR is retained. In the MTH, the abrupt switches in the AMR are much clearer, as can be seen by comparing to the original magnetoresistance traces (Fig. 1).

Figure 2 shows MTH curves for both $\rho_{\text {long }}$ and $\rho_{\text {trans }}$ for a series of magnetic field orientations $\phi$. The MTH systematically varies with $\phi$. To quantify this variation, we have evaluated the characteristic magnetic fields $H_{1}$ and $H_{2}$ (cf. Fig. 1) at which the MTH abruptly switches, and plotted them as a function of $\phi$, in Fig. 2(c). The switching fields each have one maximum within every $90^{\circ}$, and coincide once within the same interval. This "periodicity" is considered as a signature of biaxial magnetic symmetry. ${ }^{11-13}$

For the interpretation of our AMR data, we use the well established fact that the AMR effect links the resistivity of a ferromagnetic material to the orientation of the magnetization M. In thin films, the situation is most straightforward if the external magnetic field $\mathbf{H}$ is applied in the film plane. Since $\mathbf{H}, \mathbf{M}$, and $\mathbf{j}$ then all are in the film plane, the classical and the magnetization-related anomalous Hall effect vanish. ${ }^{14}$ If the magnetic structures investigated are made small enough, the AMR of one single ferromagnetic domain, ${ }^{11,12}$

$$
\begin{aligned}
& E_{\text {long }}=j \rho_{\text {long }}=j \rho_{\perp}+j\left(\rho_{\|}-\rho_{\perp}\right) \cos ^{2} \theta, \\
& E_{\text {trans }}=j \rho_{\text {trans }}=j\left(\rho_{\|}-\rho_{\perp}\right) \sin (2 \theta) / 2,
\end{aligned}
$$

becomes accessible. Here, $E_{\text {long }}$ and $E_{\text {trans }}$ are the components of the electric field along and perpendicular to $\mathbf{j}, \theta$ is the angle between $\mathbf{M}$ and $\mathbf{j}$, and $\rho_{\|}$and $\rho_{\perp}$ are the values of resistivity measured when $\mathbf{j} \| \mathbf{M}$ and $\mathbf{j} \perp \mathbf{M}$. The appearance of $E_{\text {trans }}$, Eq. (2), is only due to the tensor character of the AMR. It is often referred to as the planar Hall effect (PHE), and has been observed in conventional ferromagnetic metals, ${ }^{12,15}$ magnetic semiconductors, ${ }^{13,16}$ and in colossal magnetoresistance materials. ${ }^{17}$ However, let us emphasize that Eqs. (1) and (2) are strictly valid only for polycrystal. In a single crystal additional terms reflecting the crystal symmetry should be taken into account for a full, quantitative analysis of the AMR ${ }^{18-20}$ However, these additional terms typically are small, and thus can be omitted if only the polarity or the evolution of the AMR with magnetic field orientation is of interest. For the more qualitative analysis in the following, Eqs. (1) and (2) thus suffice.

In low external magnetic fields, the magnetization orientation is determined by the magnetic anisotropy, i.e., by the easy directions in the film plane. In a biaxial magnetic sys-
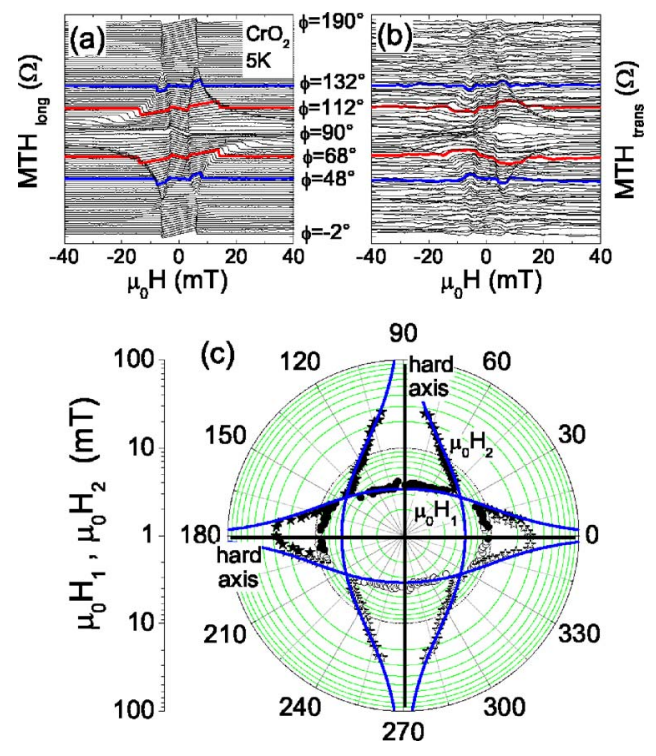

FIG. 2. (Color online) The shape of both (a) the longitudinal and (b) the transverse MTH traces characteristically depends on the orientation $\phi$ of the magnetic field. The MTH spectra given by the pairs of thicker red or blue lines are taken for the same absolute value but opposite sign of the angle $\left|90^{\circ}-\phi\right|$ between the $b$ axis and the current direction. Note also that the MTH spectra shown in panels (a) and (b) have been averaged in order to smooth the data and improve clarity. Panel (c) shows the evolution of $H_{1}$ and $H_{2}$ (see Fig. 1) with $\phi$. The full symbols represent $H_{1}$ and $H_{2}$ determined from the MTH traces in the range of $-2^{\circ}<\phi<+190^{\circ}$ accessible in our experimental setup. To visualize full $360^{\circ}$, we have again plotted these data, shifted by $180^{\circ}$, as open symbols. The full lines represent the switching fields calculated as discussed in the text. 
tem with two easy axes, the magnetization reversal process involves two subsequent switches: $\mathbf{M}$ is first aligned along one easy axis, switches to the other, and then comes back along the first easy axis with opposite orientation. In terms of Eqs. (1) and (2), the corresponding abrupt changes in $\theta$ result in two abrupt steps in both $\rho_{\text {long }}$ and $\rho_{\text {trans }}$. These switches delimit a hysteretic region in the AMR traces.

The AMR we observe in our $\mathrm{CrO}_{2}$ films (Figs. 1 and 2) indeed shows the behavior just described. Both $\rho_{\text {long }}$ and $\rho_{\text {trans }}$ enclose a hysteretic region in between two clearly visible switches at the fields $H_{1}$ and $H_{2}$. The width of this hysteretic region becomes maximal for one certain orientation $\phi$ within a $90^{\circ}$ interval in $\phi$ [Fig. 2(c)]. These angles correspond to the orientation of the hard magnetic directions. ${ }^{11-13}$ Figure 2(c) thus directly shows the presence of two hard directions within the $\mathrm{CrO}_{2}$ film plane. This observation is corroborated by comparing AMR measurements in Hall bars with the current direction either along the $\mathrm{CrO}_{2} b$ or the $c$ axis. At temperatures $1.7 \mathrm{~K} \leqslant T \leqslant 30 \mathrm{~K}$, we invariably find a behavior closely similar to the one shown in Figs. 1 and 2.

To model $H_{1}$ and $H_{2}$, we use the free energy $F=F_{\mathrm{Z}}$ $+\left(K_{\mathrm{u} 1}+K_{\sigma_{c}}-K_{\sigma_{b}}\right) \sin ^{2} \theta+K_{\sigma_{b}}+K_{\mathrm{u} 2} \sin ^{4} \theta$ of $\mathrm{CrO}_{2}{ }^{6} F_{\mathrm{Z}}$ is the Zeeman energy, $K_{\mathrm{u} 1}$ and $K_{\mathrm{u} 2}$ are the (crystalline) first and second order uniaxial anisotropy constants, respectively, $K_{\sigma_{b}}=3 \lambda \sigma_{b} / 2$ and $K_{\sigma_{c}}=3 \lambda \sigma_{c} / 2$ represent the anisotropy contributions due to epitaxial coherency strain along the crystalline $b$ and $c$ directions, ${ }^{9}$ and $\theta$ is the angle between the magnetization vector and the $\mathrm{CrO}_{2} c$ axis. The equivalence to a system with biaxial magnetic anisotropy in the film plane becomes explicitly apparent upon rewriting the $F$ as $F=F_{\mathrm{Z}}+K_{\text {ueff }} \sin ^{2} \theta+\left(K_{\mathrm{bi}} / 4\right) \sin ^{2}(2 \theta), \quad$ with $\quad K_{\text {ueff }}=K_{\mathrm{u} 1}+K_{\sigma_{c}}$ $-K_{\sigma_{b}}+K_{\mathrm{u} 2}$ and the biaxial anisotropy constant $K_{\mathrm{bi}}=-K_{\mathrm{u} 2}$. Following the approach discussed in more detail, e.g., by Cowburn et al. ${ }^{21}$ we use $F$ to derive the switching fields $H_{1,2}=\left|H_{0} \sin \left(2 \phi_{0}\right) /\left[\sin \left(\phi+\phi_{0}\right) \mp \sin \left(\phi-\phi_{0}\right)\right]\right|, \quad$ at which abrupt magnetization reorientations occur. $\phi=\phi_{0}$ is determined by $H_{1}=H_{2}=H_{0}$. As shown in Fig. 2(c), the switching fields calculated using $H_{0}=6.1 \mathrm{mT}$ and $\phi_{0}=34^{\circ}$ agree well with experiment. Moreover, $K_{\text {ueff }} / K_{\mathrm{bi}}=-\cos \left(2 \phi_{0}\right) \approx-0.37$ implies that the effective first order uniaxial anisotropy term $K_{\text {ueff }}$ in our films is smaller than the second order uniaxial anisotropy $K_{\mathrm{bi}}=-K_{\mathrm{u} 2}$. While this result might appear surprising it is fully consistent with the anisotropy constants in the literature: using $K_{\mathrm{u} 1}=44 \mathrm{~kJ} / \mathrm{m}^{3}$ and $K_{\mathrm{u} 2}=3.2 \mathrm{~kJ} / \mathrm{m}^{3}$ of bulk $\mathrm{CrO}_{2}$ at liquid $\mathrm{He}$ temperatures ${ }^{6}$ and $\sigma_{b}=9.5 \mathrm{GN} / \mathrm{m}^{2}$, $\sigma_{c}=3.7 \mathrm{GN} / \mathrm{m}^{2}$, and $\lambda=5.3 \times 10^{-6}$ of coherently strained films 9 one obtains $K_{\text {ueff }} / K_{\mathrm{bi}} \approx-0.34$. The good quantitative agreement between this value and $K_{\text {ueff }} / K_{\mathrm{bi}} \approx-0.37$ determined from the AMR experiments demonstrates that strain anisotropy qualitatively alters the magnetic anisotropy of thin $\mathrm{CrO}_{2}$ films. The resulting dominantly biaxial anisotropy in the film plane naturally explains the complex switching behavior in films of intermediate thickness $d \approx 100 \mathrm{~nm}$ reported recently, in particular, at low temperatures.

Let us come back to the planar Hall effect. The qualitative agreement between Eqs. (1) and (2) and our experiments suggests that we measure the AMR of one single domain in a biaxial magnetic energy landscape. This notion is supported by the observation that the AMR traces are fully reproducible in subsequent magnetic field sweeps. Finally, the different dependence of $\rho_{\text {long }}$ and $\rho_{\text {trans }}$ on the magnetization orientation $\left[\cos ^{2} \theta\right.$ vs $\left.\sin (2 \theta)\right]$ also is reproduced in our data, as shown explicitly by the thicker, red or blue lines in Figs. 2(a) and 2(b). The longitudinal MTH signal does not change when the magnetization reversal process goes from one easy axis to the other and back, or vice versa. In contrast, the transverse MTH trace reverses its polarity, as expected from Eq. (2). This demonstrates that $\rho_{\text {trans }}$ indeed is a true PHE and not a spurious signal. We note that the occurrence of a clear, abruptly switching PHE signal itself is a fingerprint of biaxial magnetic anisotropy.

In conclusion, we have investigated the in-plane AMR of $\mathrm{CrO}_{2}$ thin films for $1.7 \mathrm{~K} \leqslant T \leqslant 30 \mathrm{~K}$. For the low applied magnetic fields, we find two abrupt switches in both $\rho_{\text {long }}$ and $\rho_{\text {trans }}$. This observation, together with the analysis of the switching fields as a function of magnetic field orientation, demonstrates the presence of a dominant biaxial magnetic anisotropy in our $100 \mathrm{~nm}$ thick $\mathrm{CrO}_{2}$ films. Upon taking into account the magnetic anisotropy due to epitaxial coherency strain, the switching fields observed can be quantitatively modeled. This shows that crystalline strain qualitatively alters the magnetic anisotropy of $\mathrm{CrO}_{2}$ films and provides a natural explanation for the complex switching behavior reported in films of intermediate thickness.

This work is part of the research program of the "Stichting voor Fundamenteel Onderzoek der Materie (FOM)," which is financially supported by the "Nederlandse Organisatie voor Wetenschappelijk Onderzoek (NWO)." The work at the University of Alabama was supported by National Science Foundation MR-SEC Grant No. DMR0213985.

${ }^{1}$ J. M. D. Coey and M. Venkatesan, J. Appl. Phys. 91, 8345 (2002).

${ }^{2}$ J. S. Parker, P. G. Ivanov, D. M. Lind, P. Xiong, and Y. Xin, Phys. Rev. B 69, 220413 (2004).

${ }^{3}$ M. Eschrig, J. Kopu, J. C. Cuevas, and G. Schön, Phys. Rev. Lett. 90, 137003 (2003).

${ }^{4}$ R. S. Keizer, S. T. B. Goennenwein, T. M. Klapwijk, G. Miao, G. Xiao, and A. Gupta, Nature (London) 439, 825 (2006).

${ }^{5}$ S. I. Kiselev, J. C. Sankey, I. N. Krivorotov, N. C. Emley, R. J. Schoelkopf, R. A. Buhrman, and D. C. Ralph, Nature (London) 425, 380 (2003).

${ }^{6}$ D. S. Rodbell, J. Phys. Soc. Jpn. 21, 1224 (1966).

${ }^{7}$ U. Netzelmann, J. Appl. Phys. 68, 1800 (1990).

${ }^{8}$ B. Z. Rameev, A. Gupta, G. X. Miao, G. Xiao, F. Yilidz, L. R. Tagirov, and B. Aktaş, Phys. Status Solidi A 201, 3350 (2004).

${ }^{9}$ X. W. Li, A. Gupta, and G. Xiao, Appl. Phys. Lett. 75, 713 (1999).

${ }^{10}$ G. Miao, G. Xiao, and A. Gupta, Phys. Rev. B 71, 094418 (2005).

${ }^{11}$ J.-P. Jan, in Solid State Physics, edited by F. Seitz and D. Turnbull (Academic, New York, 1957), Vol. 5, pp. 1-96.

${ }^{12}$ T. R. McGuire and R. I. Potter, IEEE Trans. Magn. MAG-11, 1018 (1975).

${ }^{13}$ X. H. Tang, R. K. Kawakami, D. D. Awschalom, and M. L. Roukes, Phys. Rev. Lett. 90, 107201 (2003).

${ }^{14}$ R. C. O'Handley, Modern Magnetic Materials: Principles and Applications (Wiley, New York, 2000), pp. 557-618.

${ }^{15}$ A. Schuhl, F. Nguyen Van Dau, and J. R. Childress, Appl. Phys. Lett. 66, 2751 (1995).

${ }^{16}$ S. T. B. Goennenwein, S. Russo, A. F. Morpurgo, T. M. Klapwijk, W. Van Roy, and J. De Boeck, Phys. Rev. B 71, 193306 (2005).

${ }^{17}$ Y. Bason, L. Klein, J.-B. Yau, X. Hong, and C. H. Ahn, Appl. Phys. Lett. 84, 2593 (2004).

${ }^{18}$ W. Limmer, M. Glunk, J. Daeubler, T. Hummel, W. Schoch, R. Sauer, C. Bihler, H. Huebl, M. S. Brandt, and S. T. B. Goennenwein, Phys. Rev. B 74, 205205 (2006).

${ }^{19}$ P. K. Muduli, K.-J. Friedland, J. Herfort, H.-P. Schönherr, and K. H. Ploog, Phys. Rev. B 72, 104430 (2005).

${ }^{20}$ R. R. Birss, Symmetry and Magnetism (North-Holland, Amsterdam, 1966), pp. $1-252$.

${ }^{21}$ R. P. Cowburn, S. J. Gray, J. Ferré, J. A. C. Bland, and J. Miltat, J. Appl. Phys. 78, 7210 (1995). 